Publ. Mat. 58 (2014), 445-468

DOI: 10.5565/PUBLMAT_58214_22

\title{
EXPLICIT MINIMAL SCHERK SADDLE TOWERS OF ARBITRARY EVEN GENERA IN $\mathbb{R}^{3}$
}

\author{
A. J. Yucra Hancco*, G. A. Lobos, and V. Ramos Batista
}

\begin{abstract}
Starting from works by Scherk (1835) and by Enneper-Weierstraß (1863), new minimal surfaces with Scherk ends were found only in 1988 by Karcher (see [9, 10]). In the singly periodic case, Karcher's examples of positive genera had been unique until Traizet obtained new ones in 1996 (see [23]). However, Traizet's construction is implicit and excludes towers, namely the desingularisation of more than two concurrent planes. Then, new explicit towers were found only in 2006 by Martín and Ramos Batista (see [13]), all of them with genus one. For genus two, the first such towers were constructed in 2010 (see [22]). Back to 2009, implicit towers of arbitrary genera were found in [5]. In our present work we obtain explicit minimal Scherk saddle towers, for any given genus $2 k, k \geq 3$.
\end{abstract}

2010 Mathematics Subject Classification: 53A10.

Key words: Minimal, surfaces.

\section{Introduction}

Let $S$ be a complete minimal surface embedded in $\mathbb{R}^{3}$ and of finite total curvature. If $S$ is neither a plane nor a catenoid, the works of Schoen [21] and López-Ros [12] show that $S$ must have positive genus and a number of ends $n \leq 3$. Such an $S$ was unknown until 1984, when Costa obtained his famous example [3]. It was later generalised by Hoffman-Meeks and Hoffman-Karcher in [7] and [6], respectively. Moreover, in [7] the authors launched their conjecture that $n \leq$ genus +2 for any such $S$, which still remains open after over a quarter of a century.

In 2005, a partial answer to this conjecture was found by Meeks, Pérez, and Ros (see [14]). The authors proved that each $n \geq 2$ has an upper bound that only depends on the genus.

For $S$ embedded in a flat space, in 1989 Karcher presented several examples that answer many important questions in the Theory of Minimal Surfaces $[\mathbf{9}, \mathbf{1 0}]$. Among others, he obtained the first $S$ with positive genera and helicoidal ends, proved the existence of Schoen's surfaces [20],

*Partially supported by CAPES. 
and found singly and doubly periodic $S$ that do not belong to Scherk's minimal surface families.

By the way, Karcher constructed saddle towers $S$ of genera zero and one (in the quotient by their translation group), and number of ends $n=$ $2 k, k \geq$ genus +2 . Since then, very few new explicit $S$ were found, such as in $[\mathbf{1 3}, \mathbf{2 2}]$. This can be due to strong restrictions that underlie these surfaces.

For the genus zero case, however, a full classification was achieved in 2007. When $k=2$, Meeks and Wolf proved in [15] that $S$ belongs to Scherk's second family under the assumption of infinite symmetry. Later on, without this assumption Pérez and Traizet proved in [18] that, for any $k \geq 2, S$ must be one of the surfaces constructed by Karcher.

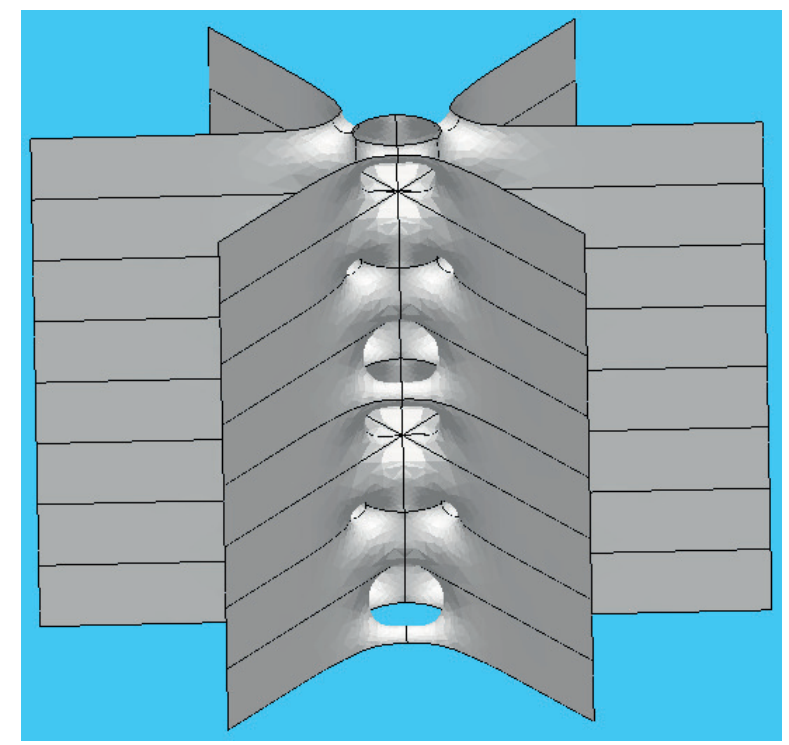

FiguRE 1. A Scherk saddle tower of genus $2 k, k=3$.

In this work we present the first explicit $S$ of arbitrary genera $2 k$ and $2 k$ Scherk ends, $k \geq 3$. More specifically, we prove

Theorem 1.1. For each natural $k \geq 3$ there exists a continuous oneparameter family of embedded minimal saddle towers in $\mathbb{R}^{3}$, of which any member $S T_{2 k}$ has its symmetry group generated by the following maps: 
1. $\pi$-rotation about the line $\left[\left(\cot \frac{\pi}{2 k}, 1,0\right)\right] \subset \mathbb{R}^{3}$;

2. reflection in the vertical plane $O x_{1} x_{3}$;

3. reflection in the horizontal plane $(0,0,1)+O x_{1} x_{2}$.

Symmetries 1 and 3 imply that $S T_{2 k}$ are invariant by the translation group $G=\langle(0,0,4)\rangle$. Moreover, $S T_{2 k} / G$ has $2 k$ Scherk ends and genus $2 k$. The surfaces $S T_{2 k}$ are embedded in $\mathbb{R}^{3}$.

Notice that items 1 and 2 make $S T_{2 k}$ invariant by $\rho$, defined as the composite of $\pi / k$-rotation around $O x_{3}$ and reflection in $O x_{1} x_{2}$. The symmetry $\rho$ will be useful in our constructions.

Regarding explicit saddle towers $S$ with arbitrary odd genus, we are convinced of their existence but we prefer to leave it as an open question, in spite of [5]. There the authors constructed implicit examples for any positive genus, for which however the inequality $n \geq 2$ (genus +2 ) does not hold. Although this could not be another kind of Hoffman-Meeks conjecture, the examples from Theorem 1.1 still verify that inequality like all explicit examples found to date.

In fact, if one aims at classifying minimal surfaces, then explicit constructions are strictly necessary.

\section{Preliminaries}

This section presents some basic definitions and theorems used throughout this work. We only consider surfaces that are regular and connected. For details see $[\mathbf{2}, \mathbf{4}, \mathbf{6}, \mathbf{8}, \mathbf{1 0}, \mathbf{1 1}, \mathbf{1 6}, \mathbf{1 7}]$.

Theorem 2.1. Let $X: R \rightarrow \mathbb{E}$ be a complete isometric immersion of a Riemann surface $R$ into a three-dimensional flat space $\mathbb{E}$. If $X$ is minimal and the total Gaussian curvature $\int_{R} K d A$ is finite, then $R$ is conformal to $\bar{R} \backslash\left\{p_{1}, p_{2}, \ldots, p_{r}\right\}$, where $\bar{R}$ is a compact Riemann surface and $r$ is a certain number of points $\left\{p_{1}, p_{2}, \ldots, p_{r}\right\} \subset \bar{R}$.

Theorem 2.2 (Weierstraß Representation). Let $R$ be a Riemann surface, $g$ and dh meromorphic function and 1-differential form on $R$, such that the zeros of dh coincide with the poles and zeros of $g$. Suppose that $X: R \rightarrow \mathbb{E}$ given by

$$
X(p):=\operatorname{Re} \int^{p} \Phi, \quad \Phi=\frac{1}{2}(1 / g-g, i / g+i g, 2) d h
$$

is well-defined. The $X$ is a conformal minimal immersion. Conversely, every conformal minimal immersion $X: R \rightarrow \mathbb{E}$ can be expressed as (1) for some meromorphic function $g$ and 1 -form $d h$. 
Definition 2.1. The pair $(g, d h)$ is the Weierstra $\beta$ data and the components of $\Phi=\left(\phi_{1}, \phi_{2}, \phi_{3}\right)$ are the Weierstraß forms on $R$ of the minimal immersion $X: R \rightarrow X(R) \subset \mathbb{E}$.

Definition 2.2. Let $R$ and $\bar{R}$ be as in Theorems 2.1 and 2.2. An end of $R$ is the image by $X$ of a punched neighbourhood $V_{p}, p \in$ $\left\{p_{1}, p_{2}, \ldots, p_{r}\right\}$, such that $\left(\left\{p_{1}, p_{2}, \ldots, p_{r}\right\} \backslash\{p\}\right) \cap \bar{V}_{p}=\emptyset$. The end is embedded if $X: V_{p} \rightarrow \mathbb{E}$ is an embedding for a sufficiently small $V_{p}$.

Theorem 2.3. Under the hypotheses of Theorems 2.1 and 2.2, the Weierstraß data $(g, d h)$ extend meromorphically on $\bar{R}$.

Theorem 2.4 (Jorge-Meeks Formula). Let $X: R \rightarrow \mathbb{E}$ be a complete minimal surface with finite total curvature $\int_{R} K d A$. If $R$ has ends that are all embedded, then $\operatorname{deg}(g)=k+r-1$, where $k$ is the genus of $\bar{R}=R \cup\left\{p_{1}, p_{2}, \ldots, p_{r}\right\}$ and $r$ is the number of ends.

Remark 2.1. In the proof of Theorem 2.4, for the case of Scherk-ends the variable $r$ counts them in pairs. The function $g$ is the stereographic projection of the Gauß map $N: R \rightarrow S^{2}$ of the minimal immersion $X$. It is a branched covering map of $\widehat{\mathbb{C}}$ and $\int_{R} K d A=-4 \pi \operatorname{deg}(g)$.

\section{Construction of the surfaces $S T_{2 k}$}

In Theorem 1.1 we denoted our surfaces by $S T_{2 k}$. This theorem is proved by Karcher's reverse construction method. Namely, we derive a list of necessary conditions that must hold in case the surfaces exist. They will end up in algebraic equations for $R, g$, and $d h$. At this point, Theorems 2.1 and 2.2 apply. Afterwards, we must prove that $X: R \rightarrow \mathbb{E}$ really corresponds to each surface $S T_{2 k}$ from Theorem 1.1.

Suppose we had a minimal surface like in Figure 1. Take the quotient by its translation group, followed by a compactification of the ends. We get a fundamental piece $\bar{S}$.

Now $\bar{S}$ has genus $2 k$, and we assume that $\bar{S}$ is invariant by $\pi / k$-rotation around $O x_{3}$ followed by a reflection in $O x_{1} x_{2}$. Let us denote this symmetry by $\rho$. Hence, the Euler characteristic of $\rho(\bar{S})$ is

$$
\chi(\rho(\bar{S}))=\frac{\chi(\bar{S})}{2 k}+\frac{2 k-1}{2 k} \cdot 2=\frac{1}{k}-2+2-\frac{1}{k}=0 .
$$

Therefore, $\rho(\bar{S})$ is a torus $T$. Due to the horizontal reflectional symmetries of $\bar{S}, T$ is a rectangular torus. 
3.1. The function $z$ on $\bar{S}$ and the Gauß map $\boldsymbol{g}(\boldsymbol{z})$. We have just obtained the rectangular torus $T=\rho(\bar{S})$. Let us now obtain two meromorphic functions $g, z$ on $\bar{S}$ through the pullback by $\rho$ of functions on $T$. Because of Remark 2.1, $g$ will be constructed by looking at the stereographic projection of the Gauß map $N: \bar{S} \rightarrow S^{2}=\widehat{\mathbb{C}}$. Regarding $z$, we choose it to make the relation $g=g(z)$ as simple as possible. See Figure 2 for an illustration.
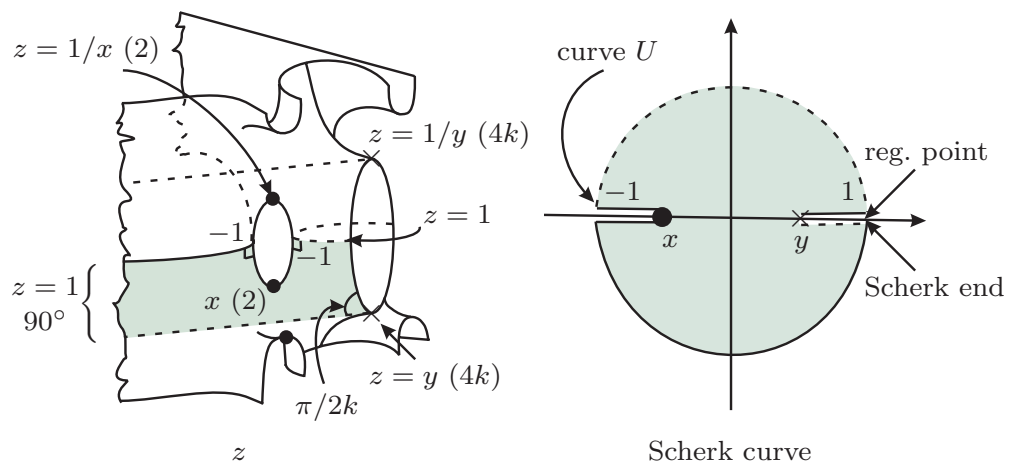

Scherk curve

Figure 2. Values of $z$ at special points of $\bar{S}$.

In $[\mathbf{1 9}]$ the author considers an elliptic function $Z^{\prime}: T \rightarrow \mathbb{C}$ as schematised in Figure 3. We define $z: \bar{S} \rightarrow \widehat{\mathbb{C}}$ as $z:=Z^{\prime} \circ \rho$.
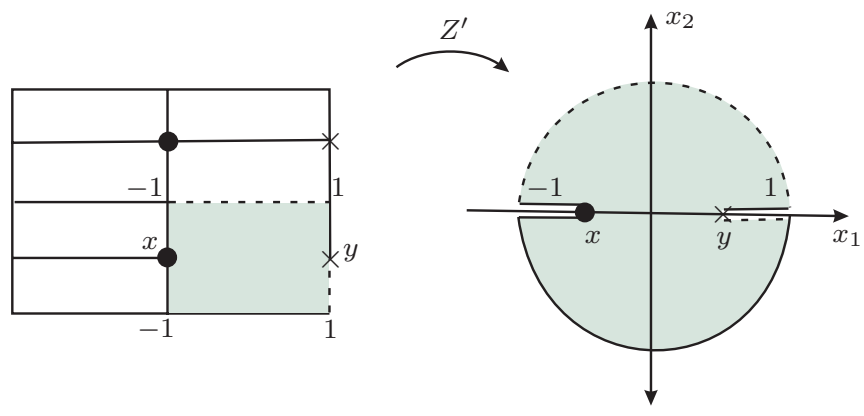

Figure 3 . The torus $T$ with some special values of $Z^{\prime}$.

By looking at the normal vector on $\bar{S}$, we know that $g$ has poles and zeros as shown in Figure 4. 


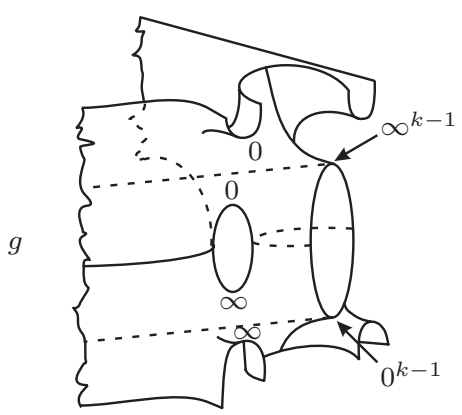

Figure 4. Poles and zeros of $g$ on $\bar{S}$.

The corresponding values of $z$ and $g$ are represented in Figures 2 and 4, including multiplicities. Therefore, we obtain the following algebraic relation between $g$ and $z$ :

$$
g^{4 k}=c\left(\frac{z-y}{1-y z}\right)^{k-1}\left(\frac{1-x z}{x-z}\right)^{2 k},
$$

where $c$ is a real constant. From Figure 2, we see that along $z(t)=e^{i \pi t}$, $0<t<1$, the following holds: $|g|=1 \Longleftrightarrow|z|=1$. Therefore,

$$
\left|g^{4 k}\right|=\left|c\left(\frac{y-z}{1-y z}\right)^{k-1}\left(\frac{1-x z}{x-z}\right)^{2 k}\right|,
$$

which implies $|c|=1$. For $z=1$ we have $g=e^{i \pi(1+k) / k}$, hence $c=1$.
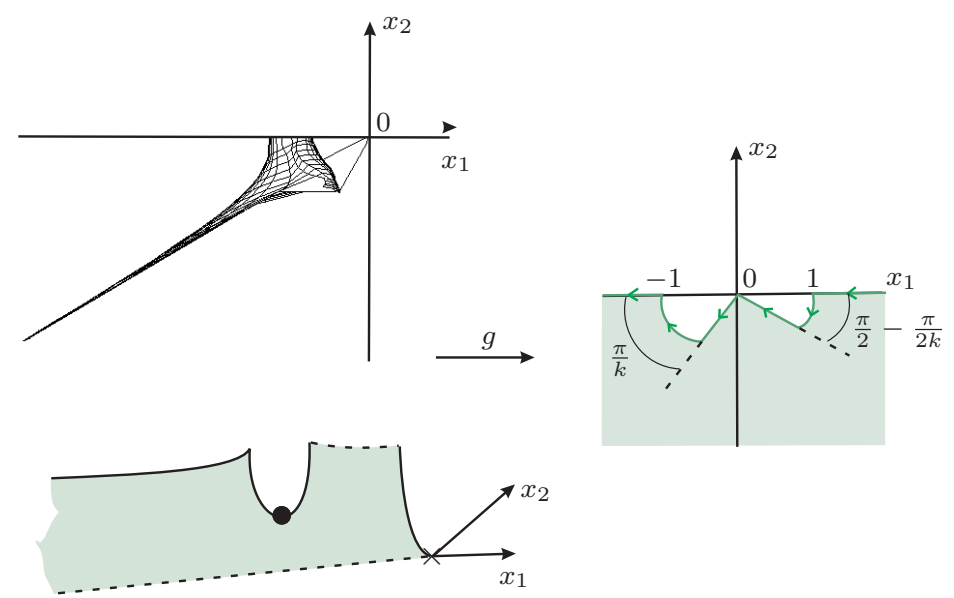

Figure 5. The fundamental domain viewed from above and the corresponding values of $g$. 
From (2), and based on Figures 2 and 5, we summarise what we know about $g$ and $d h$ in Table 1 . Of course, we have not defined $d h$ yet but it is determined by $g$, according to the theorems listed in Section 2 .

\begin{tabular}{|c||c|c|c|}
\hline stretch & $z$-values & $g$-values & $d h\left(z^{\prime}\right)$-values \\
\hline \hline 1 & $z(t)=e^{i \pi t}, 0<t<1$ & $|g|=1$ & $\in i \mathbb{R}$ \\
\hline 2 & $-1<z<x$ & $g \leq-1$ & $\notin \mathbb{R} \cup i \mathbb{R}$ \\
\hline 3 & $x<z<-1$ & $g \geq 1$ & $\notin \mathbb{R} \cup i \mathbb{R}$ \\
\hline 4 & $z(t)=e^{i \pi t},-1<t<0$ & $|g|=1$ & $\in i \mathbb{R}$ \\
\hline 5 & $1<z<y$ & $\in-i e^{.5 \pi i / k} \mathbb{R}$ & $\in i \mathbb{R}$ \\
\hline 6 & $y<z<1$ & $\in-e^{\pi i / k} \mathbb{R}$ & $\in \mathbb{R}$ \\
\hline
\end{tabular}

TABLE 1. Values of $g$ and $d h\left(z^{\prime}\right)$ along the symmetry curves.

Remark 3.1. As we shall see in Section 4, along the stretches 2 and 3 from Table $1, d h\left(z^{\prime}\right)$ takes complex values not in $\mathbb{R} \cup i \mathbb{R}$. We shall get conditions that are sufficient to prove the existence of symmetry curves. However, $S T_{2 k}$ will not have more symmetries than the ones listed in Theorem 1.1.

Table 1 shows that $g$ is consistent with the normal vector on $\bar{S}$ along special curves on the surface. Now we list important involutions of $\bar{S}$ related with the symmetries of $S T_{2 k}$ in $\mathbb{R}^{3}$. Based on (2) and Figures 2 and 5 we can summarise these involutions in Table 2:

\begin{tabular}{|c||c|c|}
\hline symmetry & involution & $g \in$ \\
\hline \hline$z(t)=e^{i \pi t}, 0<t<1$ & $(z, g) \rightarrow(1 / \bar{z}, 1 / \bar{g})$ & $S^{1}$ \\
\hline$-1<z<x$ & $(z, g) \rightarrow(\bar{z}, \bar{g})$ & $(-\infty,-1)$ \\
\hline$x<z<-1$ & $(z, g) \rightarrow(\bar{z}, \bar{g})$ & $(1, \infty)$ \\
\hline$z(t)=e^{i \pi t},-1<t<0$ & $(z, g) \rightarrow(1 / \bar{z}, 1 / \bar{g})$ & $S^{1}$ \\
\hline $1<z<y$ & $(z, g) \rightarrow\left(\bar{z},-e^{i \pi / k} \bar{g}\right)$ & $-i e^{.5 i \pi / k} \mathbb{R}$ \\
\hline$y<z<1$ & $(z, g) \rightarrow\left(\bar{z}, e^{2 i \pi / k} \bar{g}\right)$ & $-e^{i \pi / k} \mathbb{R}$ \\
\hline
\end{tabular}

TABLE 2. Involutions on $\bar{S}$. 


\section{The differential $d h$ in terms of $z$}

Since $S T_{2 k}$ has Scherk-ends, their corresponding points of $\bar{S}$ are exactly the poles of $d h$. Regarding the zeros of $d h$, they coincide with the points of $\bar{S}$ at which $g=0$ or $g=\infty$, including multiplicity. We shall have to read off information about $d z$ in order to write down an equation for $d h$.

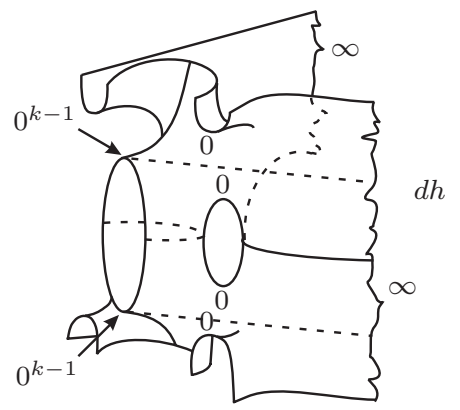

Figure 6. Poles and zeros of $d h$ on $\bar{S}$.

Figure 2 shows two points marked with $\times$ at which $z=y$ and $z=1 / y$. There we have $d z=0$ of order $4 k-1$. Moreover, at $z=x$ we have $d z=0$ of order 1. Now Figure 7 illustrates the divisor of $d h$.

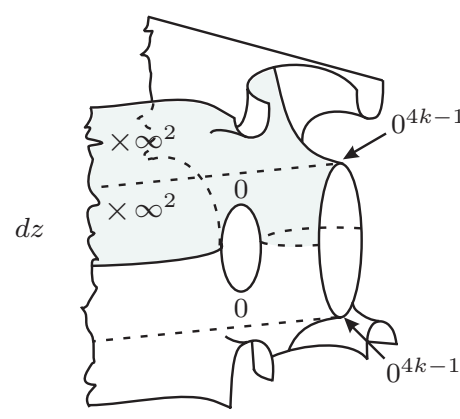

(a)

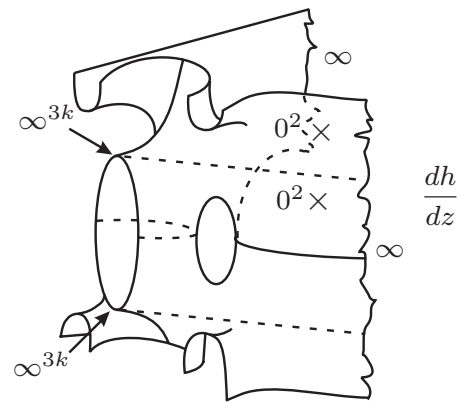

(b)

Figure 7. (a) Divisor of $d z$ on $\bar{S}$; (b) divisor of $d h / d z$ on $\bar{S}$.

In order to obtain $d h$ by means of $d z$, we must analyse the divisor of $f:=(1-y z)(y-z)$. According to Figure $7(\mathrm{a})$, it is sufficient to construct $F: \bar{S} \rightarrow \widehat{\mathbb{C}}$ such that $F=f \cdot d h / d z$. Once we have $F$, then

$$
d h=F d z / f .
$$


Notice that there are distinct points of $\bar{S}$ at which $z$ takes the same value 1 , namely at the ends and at certain regular points. Since $d h$ has no poles except for the ends, then we shall have to introduce new functions besides $z$ and $g$. They are depicted in Figure 9. In this figure

$$
v_{2}=1-\frac{i}{v_{1}}\left(\frac{1-y}{1+y}\right) \text {, }
$$

where

$$
v_{1}:=\sqrt{\left(\frac{1-x}{1+x}\right)^{2}-\left(\frac{1-y}{1+y}\right)^{2}} .
$$

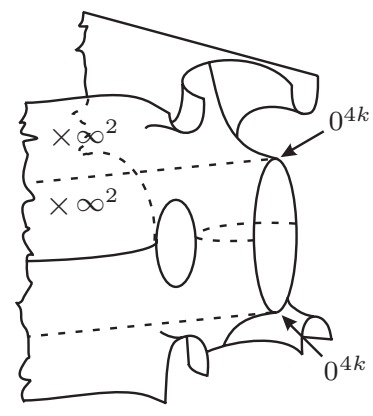

(a)

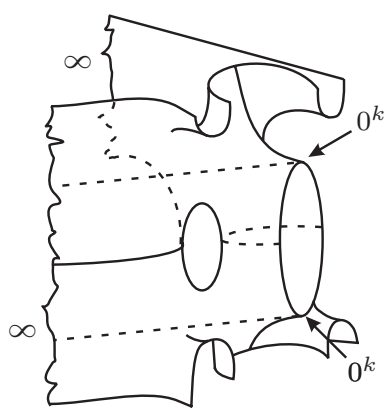

(b)

Figure 8. (a) Values of $f$; (b) values of $f \cdot d h / d z$ on $\bar{S}$.

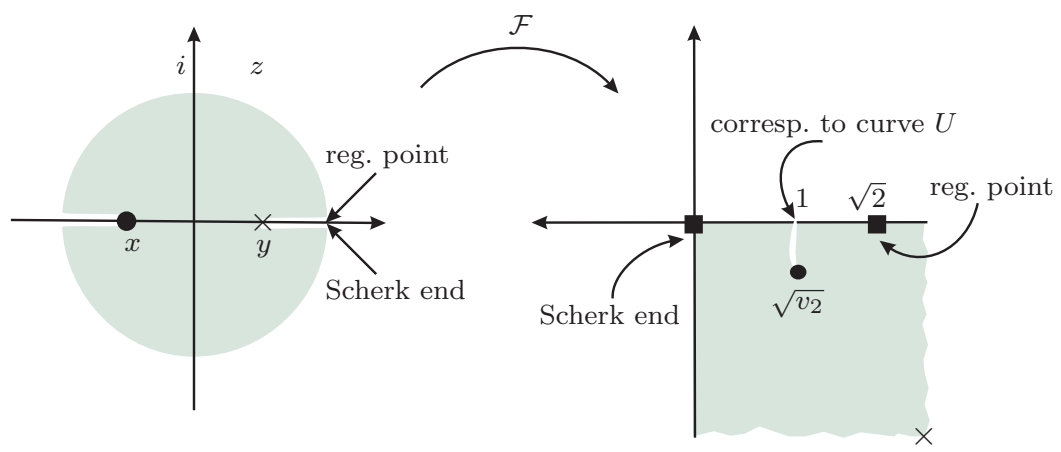

Figure 9. The function $\mathcal{F}=\sqrt{1-\frac{i}{w}\left(\frac{1-y}{1+y}\right)}$. 
Now

$$
w:=\sqrt{\left(\frac{1-z}{1+z}\right)^{2}-\left(\frac{1-y}{1+y}\right)^{2}},
$$

is well-defined in a branched covering of $\bar{S}$ that we call $\bar{R}$. The algebraic equation of $\bar{R}$ will be discussed later on. This way we get

$$
\mathcal{F}=\sqrt{1-\frac{i}{w}\left(\frac{1-y}{1+y}\right)} .
$$

Therefore, $\mathcal{F} \cdot f \cdot d h / d z$ has neither poles nor zeros, whence must be a non-zero complex constant c. Namely,

$$
d h=\mathbf{c} \cdot F d z / f,
$$

where $F:=1 / \mathcal{F}$. Now we show that $\mathbf{c}=1$. Indeed, since stretch 5 in Table 1 is represented by a straight line, the 3rd coordinate of (1) must be zero. But along this stretch we have $F d z / f \in i \mathbb{R}$, and in (1) we compute the real part of a complex integral. Hence $\mathbf{c}$ must be real. The property of a surface being minimal in $\mathbb{R}^{3}$ is preserved by the antipodal map and by homotheties. Therefore we can take $\mathbf{c}=1$.

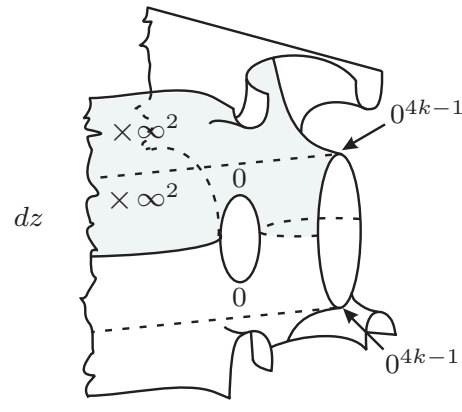

(a)

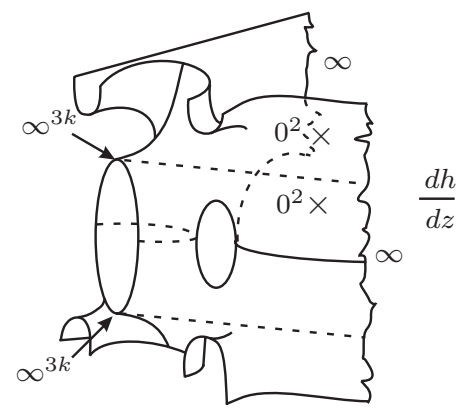

(b)

Figure 10. Values of $\mathcal{F}$ on $\bar{S}$.

According to Theorems 2.1 and 2.2 , dh can be defined on $\bar{S}$ by a rational function involving only $g, d g, z$, and $d z$. However, its formula is probably far too extensive. Then we use square roots as explained above. Of course, they are not well-defined on $\bar{S}$, but on a branched covering that we call $\bar{R}$. In order to describe $\bar{R}$ by an algebraic equation, we consider

$$
\mathcal{F}=\sqrt{1-\frac{i}{w}\left(\frac{1-y}{1+y}\right)}
$$


and

whence

$$
w^{2}=\frac{4 f}{(1+z)^{2}(1+y)^{2}},
$$

$$
\left(\mathcal{F}^{2}-1\right)^{2}=-\frac{1}{4} \frac{(1+z)^{2}(1-y)^{2}}{(y-z)(1-y z)} .
$$

This way we get the polynomial

$$
a z^{2}+b z+c=0
$$

where $a, b$, and $c$ depend on $\mathcal{F}$ and on some complex constants. This results in

$$
z=\frac{-b \pm \sqrt{\Delta}}{2 a} .
$$

Now (2) can be rewritten as

$$
A_{3 k-1}(g) \cdot z^{3 k-1}+\cdots+A_{0}(g)=0,
$$

where $A_{j}$ is a polynomial in $g, \forall j$. By applying (4) to (5) we get

$$
\pm \sqrt{\Delta} \cdot E_{1}=E_{2},
$$

where $E_{1}$ and $E_{2}$ are polynomials in $g$ and $\mathcal{F}$. We square both sides of $(6)$ and finally get a polynomial $P(g, \mathcal{F})=0$, which gives an algebraic equation for $\bar{R}$. The functions $g$ and $\mathcal{F}$ are then well-defined on $\bar{R}$. Of course, there is a projection $B: \bar{R} \rightarrow \bar{S}$ given by $B(g, \mathcal{F})=g$. However, there is no projection that makes $\mathcal{F}$ well-defined on $\bar{S}$, since we must use square roots to equate $\mathcal{F}$ on $\bar{S}$.

We recall (3) and see that $F / f$ is a rational function on $\bar{R}$. However, all computations there would have to match the computations on $\bar{S}$ that use square roots, because $\bar{R}$ was obtained from them. Therefore, we shall keep on working with the square roots.

Now we can analyse $d h$ along the symmetry curves in Table 1 . Observe that

$$
d h=\frac{z}{f} \cdot \frac{1}{\mathcal{F}} \cdot \frac{d z}{z} .
$$

On the stretch $y<z<1$ the function $\mathcal{F}$ is real and positive, whereas $f$ is real and negative. Since the curve is $z(t)=t$, we have $d h\left(z^{\prime}\right) \in \mathbb{R}$. For $1<z<y,-i \mathcal{F}$ is real and negative, thus $d h\left(z^{\prime}\right) \in i \mathbb{R}$.

Regarding stretches 1 and 4, there we have $z(t)=e^{i \pi t}, 0<t<1$, and $-1<t<0$, respectively. Notice that $z / f=y^{-1} /(1 / z+z-(1 / y+y))$ and on these stretches $z / f$ and $\mathcal{F}$ are both real. Therefore, $d h\left(z^{\prime}\right) \in i \mathbb{R}$ because $d z / z \in i \mathbb{R}$. 
From Table 1 we see that the $z$-curves 2 and 3 are geodesics. Moreover, the geodesics are planar curves in cases 1,4 , and 6 because $\left.(d h \cdot d g / g)\right|_{z^{\prime}} \in$ $\mathbb{R}$, and a straight line in case 5 because $\left.(d h \cdot d g / g)\right|_{z^{\prime}} \in i \mathbb{R}$. Therefore, our minimal surfaces $S T_{2 k}$ are symmetric with respect to $1,4,5$, and 6 .

But we recall Remark 3.1 regarding curves 2 and 3. From Table 1, $d h\left(z^{\prime}\right) \notin \mathbb{R} \cup i \mathbb{R}$. Indeed, for $z(t)=t,-1<t<x$, we have $z / f \in \mathbb{R}$ and $\mathcal{F} \notin \mathbb{R} \cup i \mathbb{R}$. Hence $d h\left(z^{\prime}\right) \notin \mathbb{R} \cup i \mathbb{R}$. The surfaces $S T_{2 k}$ are not symmetric with respect to the curves 2 and 3 , as we shall prove later.

\section{The period problem}

Figure 5 shows the fundamental domain of $S T_{2 k}$. Some important details are reproduced again in Figure 11(a), but there we indicate a path that begins at a point marked with $\times$. The path goes upwards and then from the right to the left-hand side, where we find its end. If the $\times$-point is the origin of $\mathbb{R}^{3}$, then the end of the path ought to be in the plane $x_{2}=0$. This path is what we call a period curve.

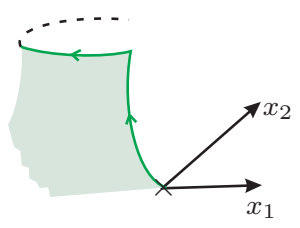

(a)

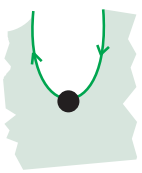

(b)

Figure 11. Period curves.

Another one is the $U$-curve in Figure 11(b). Its period is zero if it has both extremes at the same height. This is determined by the integral of $d h$ alone, and $d h$ is given by square roots. What happens is that their signs change at the vertex of the $U$-curve, marked with a bullet in Figure 11. The change of sign automatically implies that both extremes of the $U$-curve do attain the same height.

We recall that $d h$ has an algebraic expression in $\bar{S}$ involving $(z, g, d z, d g)$. This is ensured by Theorems 2.1 and 2.2. Since $d h=$ $d h(z, g, d z, d g)$ on $\bar{S}$ is the general expression, it involves extra complex parameters. For the $U$-curve, its extremes will have heights that depend on these parameters, and the heights will not coincide in general. However, by using square roots in the local expression of $d h$ on $\bar{S}$, the extra parameters are forced to assume constant values. In fact, we do not even discuss them because of the straight choice of (3). But there still remain 
the two free parameters $x$ and $y$, which also take part in the algebraic equation of $\bar{R}$.

Hence, we only have one period problem. For convenience of the reader, herewith we reproduce the Weierstraß data:

$$
\begin{gathered}
g^{4 k}=(-1)^{k-1}\left(\frac{y-z}{1-y z}\right)^{k-1}\left(\frac{1-x z}{x-z}\right)^{2 k}, \\
d h=\frac{1}{\sqrt{1-\frac{i(1-y)}{w(1+y)}}} \frac{d z}{(1-y z)(y-z)},
\end{gathered}
$$

where

$$
w=\sqrt{\left(\frac{1-z}{1+z}\right)^{2}-\left(\frac{1-y}{1+y}\right)^{2}} .
$$

Let us now analyse some special stretches depicted in Figure 11(a). According to the branches of square root that we have chosen, $1=$ $e^{4(k+1) \pi i}$ for $z(t)=t, y \leq t \leq 1$, which is the upward stretch from $\times$ to a point in $z^{-1}(1)$. Hence

$$
g=-e^{i \pi / k}\left(\frac{t-y}{1-y t}\right)^{\frac{k-1}{4 k}}\left(\frac{1-x t}{t-x}\right)^{\frac{1}{2}} .
$$

Along the stretch $z(t)=e^{i t}, 0 \leq t \leq \pi$, we have

$$
g=-e^{\frac{i \pi}{k}} \cdot e^{\frac{i(k+1) t}{4 k}}\left(\frac{y-e^{i t}}{y-e^{-i t}}\right)^{\frac{k-1}{4 k}}\left(\frac{x-e^{-i t}}{x-e^{i t}}\right)^{\frac{1}{2}} .
$$

Now we analyse $w$ more carefully along $z(t)=t, y \leq t \leq 1$. From (10) we have

$$
w=-\frac{2 i \sqrt{(1-y t)(t-y)}}{(1+t)(1+y)} .
$$

Let us define $Y:=i(1-y) /(1+y)$. Hence

$$
\frac{Y}{w}=-\frac{1}{2} \frac{(1+t)(1-y)}{\sqrt{(1-y t)(t-y)}}
$$

and therefore

Finally,

$$
d h=\frac{1}{\left\{[(1-y t)(t-y)]^{1 / 2}+(1+t)(1-y) / 2\right\}^{1 / 2}} \cdot \frac{d t}{[(1-y t)(t-y)]^{3 / 4}}
$$


In general, (10) rewrites as

$$
\frac{w}{Y}=-\frac{2 i \sqrt{(1-y z)(z-y)}}{(1+z)(1+y)} \frac{(1+y)}{i(1-y)}=-\frac{2 \sqrt{y} \sqrt{(1 / y+y)-(1 / z+z)}}{\sqrt{z}(1 / z+1)(1-y)},
$$

and

$$
\begin{aligned}
d h & =\frac{1 / y}{\sqrt{1-\frac{i(1-y)}{w(1+y)}}} \frac{d z / z}{(1 / y-z)(y / z-1)} \\
& =\frac{1 / y}{\sqrt{1-\frac{i(1-y)}{w(1+y)}}} \frac{d z / z}{(1 / z+z)-(1 / y+y)} .
\end{aligned}
$$

For $z(t)=e^{i t}, 0 \leq t \leq \pi$, this results in

$$
\begin{aligned}
\frac{w}{Y} & =-\frac{\sqrt{y} \sqrt{-2 \cos t+(1 / y+y)}}{(1-y) \cos (t / 2)} \Rightarrow \sqrt{1-\frac{Y}{w}} \\
& =\sqrt{1+\frac{(1-y)(\cos (t / 2))}{\sqrt{y}(1 / y+y-2 \cos t)^{1 / 2}}}
\end{aligned}
$$

Hence

$$
d h=\frac{i / y}{\sqrt{1+\frac{(1-y) \cos (t / 2)}{\sqrt{y}(1 / y+y-2 \cos t)^{1 / 2}}}} \frac{d t}{2 \cos t-(1 / y+y)} .
$$

Associated with $z(t)=t, y \leq t \leq 1$, we have

$$
I_{1}^{k}=\operatorname{Re} \int_{y}^{1} \phi_{2} d h=\operatorname{Re} \int_{y}^{1}\left(\frac{i}{g}+i g\right) d h .
$$

From (11), (13), and the change of variables $t=y+s^{4 k}$ we have

$$
\begin{aligned}
\left.I_{1}^{k}\right|_{(y, x)}= & \operatorname{Re} i \int_{0}^{(1-y)^{1 / 4 k}}\left\{\left[-e^{-i \pi / k}\left(\frac{1}{1-y^{2}-s^{4 k} y}\right)^{-\frac{k-1}{4 k}}\left(\frac{1-x\left(y+s^{4 k}\right)}{\left(y+s^{4 k}\right)-x}\right)^{-1 / 2}\right.\right. \\
& \left.-e^{i \pi / k} s^{2(k-1)}\left(\frac{1}{1-y^{2}-s^{4 k} y}\right)^{\frac{k-1}{4 k}}\left(\frac{1-x\left(y+s^{4 k}\right)}{\left(y+s^{4 k}\right)-x}\right)^{1 / 2}\right] \\
& \left.\times \frac{1}{\sqrt{\sqrt{\left(1-y^{2}-s^{4 k} y\right)\left(s^{4 k}\right)}+\frac{\left(1+y+s^{4 k}\right)(1-y)}{2}}} \frac{4 k d s}{\sqrt[4]{\left[\left(1-y^{2}-s^{4 k} y\right)\right]^{3}}}\right\} .
\end{aligned}
$$


We recall that $y \in(0,1)$ and $x \in(-1,0)$. For $(y, x) \rightarrow(0,-1)$ we have

(16) $\left.\quad I_{1}^{k}\right|_{(0,-1)}=\operatorname{Re}\left\{4 k \sqrt{2} i \int_{0}^{1}\left[-e^{-i \pi / k}-e^{i \pi / k} s^{2(k-1)}\right] \frac{d s}{1+s^{2 k}}\right\}$.

Let us observe what happens to (16) when $k=3$ :

$$
\left.I_{1}^{k}\right|_{(0,-1)}=\operatorname{Re}\left\{12 \sqrt{2} i\left[-e^{-i \pi / 3} \int_{0}^{1} \frac{d s}{1+s^{6}}-e^{i \pi / 3} \int_{0}^{1} \frac{s^{4} d s}{1+s^{6}}\right]\right\} .
$$

Since

$$
\begin{aligned}
\int \frac{d s}{1+s^{6}}=\frac{1}{12}( & -\sqrt{3} \ln \left(s^{2}-\sqrt{3} s+1\right)+\sqrt{3} \ln \left(s^{2}+\sqrt{3} s+1\right) \\
& -2 \arctan (\sqrt{3}-2 s)+4 \arctan (s)+2 \arctan (2 s+\sqrt{3})), \\
\int \frac{s^{4} d s}{1+s^{6}}=\frac{1}{12}( & \sqrt{3} \ln \left(s^{2}-\sqrt{3} s+1\right)-\sqrt{3} \ln \left(s^{2}+\sqrt{3} s+1\right) \\
& -2 \arctan (\sqrt{3}-2 s)+4 \arctan (s)+2 \arctan (2 s+\sqrt{3})),
\end{aligned}
$$

then

$$
\begin{aligned}
\left.I_{1}^{k}\right|_{(0,-1)} & \approx \operatorname{Re}\left\{i 12 \sqrt{2}\left[e^{-i \pi / 3}(0.90377)+e^{i \pi / 3}(0.14343)\right]\right\}, \\
\left.I_{1}^{k}\right|_{(0,-1)} & \approx-11.17447 .
\end{aligned}
$$

Now we compute $I_{1}^{k}$ for $(y, x) \rightarrow(0,0)$ :

(17) $\left.I_{1}^{k}\right|_{(0,0)}=\operatorname{Re}\left\{i 4 k \sqrt{2} \int_{0}^{1}\left[-e^{-i \pi / k} s^{2 k}-e^{i \pi / k} s^{-2}\right] \frac{d s}{1+s^{2 k}}\right\}=+\infty$.

Hence $-\left.I_{1}^{k}\right|_{(0,0)}=-\infty \forall k$. For the horizontal path depicted in Figure $13(\mathrm{a})$ we have

$$
I_{2}^{k}=\operatorname{Re} \int_{\alpha} \phi_{2} d h=\operatorname{Re} \int_{\alpha}\left(\frac{i}{g}+i g\right) d h
$$


where $\alpha(t)=e^{i t}$ with $0 \leq t \leq \pi$. From (12) and (14) we have

$$
\begin{aligned}
I_{2}^{k}=\operatorname{Re} i \int_{0}^{\pi}\{ & {\left[-e^{\frac{-i \pi}{k}} e^{-\frac{i(k+1) t}{4 k}}\left(\frac{y-e^{i t}}{y-e^{-i t}}\right)^{-\frac{k-1}{4 k}}\left(\frac{x-e^{-i t}}{x-e^{i t}}\right)^{-\frac{1}{2}}\right.} \\
& \left.-e^{\frac{i \pi}{k}} e^{\frac{i(k+1) t}{4 k}}\left(\frac{y-e^{i t}}{y-e^{-i t}}\right)^{\frac{k-1}{4 k}}\left(\frac{x-e^{-i t}}{x-e^{i t}}\right)^{\frac{1}{2}}\right] \\
& \left.\times \frac{i / y}{\sqrt{1+\frac{(1-y) \cos (t / 2)}{\sqrt{y}(1 / y+y-2 \cos t)^{1 / 2}}}} \frac{d t}{2 \cos t-(1 / y+y)}\right\} .
\end{aligned}
$$

We begin with $I_{2}^{k}$ and make $(y, x) \rightarrow(0,-1)$. This results in

$$
\left.I_{2}^{k}\right|_{(0,-1)}=-2 \operatorname{Re}\left\{\int_{0}^{\pi} \cos \left(\frac{4 \pi+t(k-1)}{4 k}\right) \frac{d t}{\sqrt{1+\cos (t / 2)}}\right\}
$$

For $k=3$, (20) becomes

$$
\left.I_{2}^{3}\right|_{(0,-1)}=-2 \operatorname{Re}\left\{\int_{0}^{\pi} \cos \left(\frac{2 \pi+t}{6}\right) \frac{d t}{\sqrt{1+\cos (t / 2)}}\right\} .
$$

Since $0 \leq t \leq \pi,(21)$ rewrites as

$$
-2 \leq-2 \cos \left(\frac{2 \pi+t}{6}\right) \frac{1}{\sqrt{1+\cos (t / 2)}} \leq 2,
$$

whence

$$
-2 \pi \leq\left. I_{2}^{3}\right|_{(0,-1)} \leq 2 \pi \text {. }
$$

Finally, we analyse $I_{2}^{k}$ when $(y, x) \rightarrow(0,0)$. From (19) it follows that

$$
\left.I_{2}^{k}\right|_{(0,0)}=-\operatorname{Re} 2 \int_{0}^{\pi} \cos \left(\frac{4 \pi-t(k+1)}{4 k}\right) \frac{d t}{\sqrt{1+\cos (t / 2)}} .
$$

For $k=3,(22)$ rewrites as

$$
\left.I_{2}^{3}\right|_{(0,0)}=-\operatorname{Re} 2 \int_{0}^{\pi} \cos \left(\frac{\pi-t}{3}\right) \frac{d t}{\sqrt{1+\cos (t / 2)}} .
$$

Since $0 \leq t \leq \pi$, then

$$
-2 \leq-2 \cos \left(\frac{\pi-t}{3}\right) \frac{1}{\sqrt{1+\cos (t / 2)}} \leq 2
$$

whence

$$
-2 \pi \leq\left. I_{2}^{3}\right|_{(0,0)} \leq 2 \pi .
$$


Therefore,

$$
\begin{aligned}
& \left.I_{1}^{3}\right|_{(0,-1)} \approx-11.1747, \\
& -2 \pi \leq\left. I_{2}^{3}\right|_{(0,-1)} \leq 2 \pi,
\end{aligned}
$$

and then $-I_{1}^{3}>I_{2}^{3}$ at $(0,-1)$. Now

$$
\begin{gathered}
-\left.I_{1}^{3}\right|_{(0,0)}=-\infty, \\
-2 \pi \leq\left. I_{2}^{3}\right|_{(0,0)} \leq 2 \pi,
\end{gathered}
$$

whence $-I_{1}^{3}<I_{2}^{3}$ at $(0,0)$. By the Intermediate Value Theorem, there exists a point $\left(y^{*}, x^{*}\right)$ at which $-I_{1}^{3}=I_{2}^{3}$. We are ready to prove the following result, which concludes this section:

Lemma 5.1. For any natural $k \geq 3$ there exists a point at which $-I_{1}^{k}=$ $I_{2}^{k}$.

Proof: From (16), $-\left.I_{1}^{k}\right|_{(0,-1)}$ will be increasing with $k$ exactly when $\pi \cdot \frac{\sin (\pi / k)}{(\pi / k)} \cdot\left(-s^{-2}+\frac{1+s^{-2}}{1+s^{2 k}}\right)$ is increasing with $k$. But this is obvious to the function $\frac{\sin (\pi / k)}{(\pi / k)}$, and also to the function $-s^{-2}+\frac{1+s^{-2}}{1+s^{2 k}}$. Since both are positive, then $-\left.I_{1}^{k}\right|_{(0,-1)}$ is increasing with $k$. Moreover, for all $k \geq 3$ we have

$$
\begin{aligned}
& -2 \pi \leq\left. I_{2}^{k}\right|_{(0,-1)} \leq 2 \pi, \\
& -2 \pi \leq\left. I_{2}^{k}\right|_{(0,0)} \leq 2 \pi,
\end{aligned}
$$

because these inequalities hold for $k=3$, and the same computations lead to the general case. Hence $-\left.I_{1}^{k}\right|_{(0,-1)} \geq-\left.I_{1}^{3}\right|_{(0,-1)}>\left.I_{2}^{k}\right|_{(0,-1)}$, and also $-\left.I_{1}^{k}\right|_{(0,0)}=-\infty<\left.I_{2}^{k}\right|_{(0,0)}$.

We conclude this section by showing that the $S T_{2 k}$ form a continuous one-parameter family of surfaces. Of course, our $S T_{2 k}$ are given by the Weierstraß data (8) and (9), both defined on $\bar{S} \backslash\left\{p_{1}, \ldots, p_{2 k}\right\}$. The algebraic equation of $\bar{S}$ is again (8), and $p_{j}$ are exactly the poles of $d h$, $j=1, \ldots, 2 k$. Moreover, $(y, x)$ are the points that Lemma 5.1 refers to.

Fix $k \geq 3$ and a point $\left(y^{*}, x^{*}\right)$ at which $-I_{1}^{k}=I_{2}^{k}$. Notice that $I_{1}^{k}$ and $I_{2}^{k}$ are both real analytic functions of $(y, x)$. According to the proof of Lemma 5.1, we can take $\left(y^{*}, x^{*}\right)$ as a point obtained by a crossing of their graphs, which is transversal up to arbitrarily small perturbations. Now standard arguments from Differential Topology show that $\left(y^{*}, x^{*}\right)$ must belong to a whole solution curve $\mathcal{C}$ in the triangular domain $\{(y, x) \mid$ 
$-1<x<y<1\}$. We can parametrise $\mathcal{C}$ as $(y(t), x(t))$, and then $t$ parametrises $S T_{2 k}$ as well.

Such arguments were already detailed in other works, and so we shall skip them here. See, for instance [1, pp. 101-102].

\section{Embeddedness}

We recapitulate that the compact Riemann surface $\bar{S}$ is given by (2), where $(x, y)$ is the point that Lemma 5.1 refers to. If $B \subset \bar{S}$ denotes the branch points of $g$, then the ends of $\bar{S}$ are $\left\{p_{1}, \ldots, p_{2 k}\right\}=z^{-1}(1) \backslash B$, as shown in the next subsection. We have $d h$ defined on $\bar{S}$, with a local expression given by (3). From Theorem 2.2, this defines a minimal immersion $X: R \rightarrow \mathbb{E}$, where $R=\bar{S} \backslash\left\{p_{1}, \ldots, p_{2 k}\right\}$. The purpose of this section is to prove that $X$ is an embedding.

6.1. Poles and zeros of $\boldsymbol{d g}$. We know that $\operatorname{deg}(d g)=-\chi_{\bar{S}}=4 k-2$. According to Figure $4, d g$ should have exactly $k+(2+2) k=5 k$ poles. Notice that the multiplicity is always included in our analysis.

Since $\operatorname{deg}(d g)=\operatorname{Nr} z \operatorname{zeros}(g)-\operatorname{Nr} \operatorname{poles}(g)$, then $d g$ has exactly $9 k-$ 2 zeros. Now we are going to locate these zeros by geometric arguments. They can be checked analytically from (2).

According to Figure $4, k-2$ of the zeros are at the saddle point marked with $\times$ in Figure 2 where $z=y$. We have $k 4$-fold saddle points along each horizontal closed curve of symmetry, which gives a subtotal of $2 k$ points. Each of them adds 2 zeros for $d g$. Hitherto we have $5 k-2$ zeros.

Somewhere along each ray that departs from the $\times$-point, the unitary normal has an inflexion, hence $d g=0$ there. By counting all such rays, at the top and at the bottom of the fundamental piece, we arrive at the $4 k$ remaining zeros that finally totalize $9 k-2$.

6.2. The $U$-curve. Soon we shall see that the $U$-curve is not of symmetry. However, in Section 4 we explained that our $d h$ is a particular case of a more general formula that should involve $z, d z, g$, and $d g$. Therefore, it is highly possible that, for each $k$, the surfaces $S T_{2 k}$ are representatives of a continuous two-parameter family of surfaces. This family is very likely to have more symmetric members for which the $U$-curve $i s$ of symmetry.

Therefore, herewith we present arguments under this assumption. It will be slacked later, but the reader will notice how simple the arguments are in this case. Most of these arguments remain valid in the slacked case, and so we ease the understanding of our proof. 
We named $P$ the fundamental piece of $S T_{2 k}$. In Figure 13, the shaded region represents a fundamental domain of $P$.

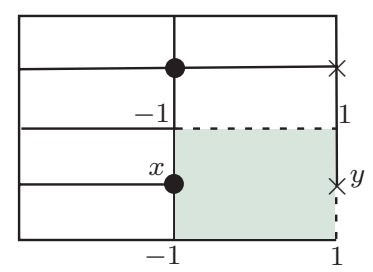

Figure 12. A fundamental domain in the torus $T$.

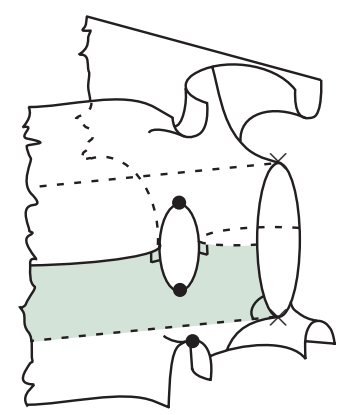

Figure 13. A fundamental domain of $P$.

Let $\mathcal{R}$ be the circle in Figure 14 left. The image $\mathcal{D}=g(\mathcal{R})$ is depicted in Figure 14 right. It is contained in a hemisphere of $S^{2}=\widehat{\mathbb{C}}$. Hence, there is a direction in which the orthogonal projection of $X(\mathcal{R})$ is an immersion. For instance, direction $O x_{2}$. This way $\left(x_{1}, x_{3}\right): \mathcal{R} \rightarrow \mathbb{R}^{2}$ is an immersion when restricted to the interior of $\mathcal{R}$. The image $\left(x_{1}, x_{3}\right)(\mathcal{R}) \subset$ $\mathbb{R}^{2}$ has one out of four basic features described in Figure 15.

If the $U$-curve is planar, then it is convex. Indeed, the Gaussian curvature

$$
K=-\left(\frac{2}{|g|+1 /|g|}\right)^{4}\left|\frac{d g / g}{d h}\right|^{2}
$$

vanishes on $U$ exactly where $d g=0$ there. But in Subsection 6.1 we saw that this never happens. 

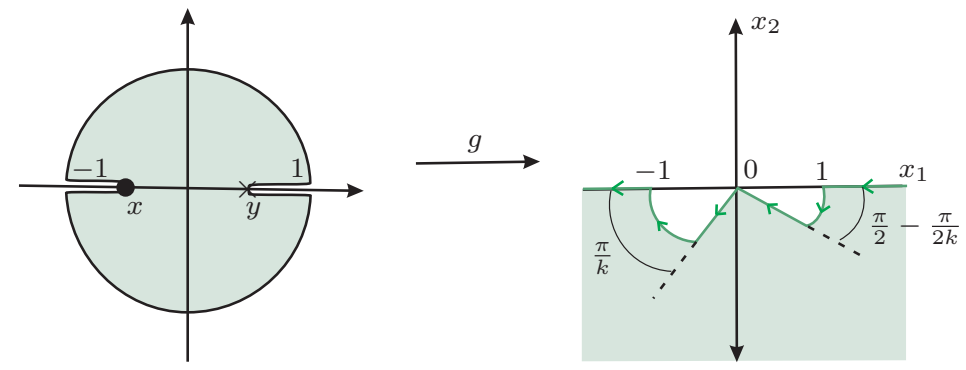

Figure 14. Image $\mathcal{D}=g(\mathcal{R})$.

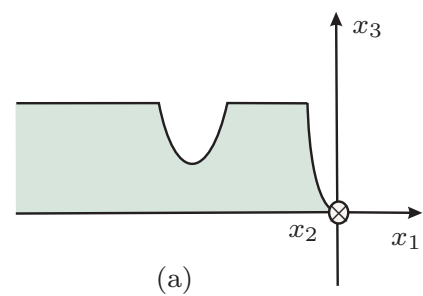

(a)

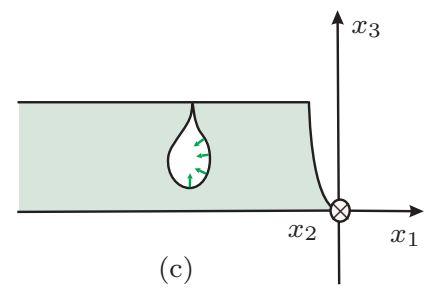

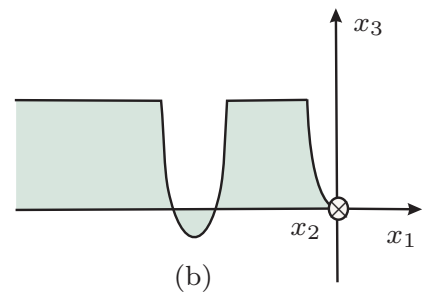

(b)

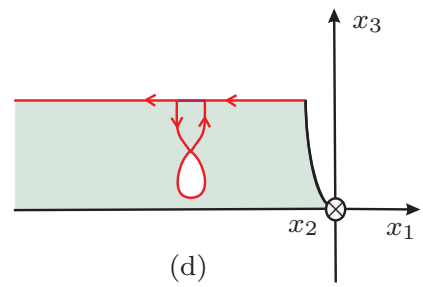

FiguRE 15. Four basic features of $\left(x_{1}, x_{3}\right)(\mathcal{R})$.

Since $\left.\left(x_{1}, x_{3}\right)\right|_{\mathcal{R}}$ is an immersion, it is open and continuous. Hence, $\left(x_{1}, x_{3}\right)(\mathcal{R})$ is an open connected subset of $\mathbb{R}^{2}$, which discards Figure 15(b). Figures 15(c) and 15(d) are also discarded because $g$ is injective along $U$. We finally remain with Figure 15(a).

Let $G$ be the interior of $\left(x_{1}, x_{3}\right)(\mathcal{R})$, which is simply connected. Of course, $\mathcal{R} \subset \widehat{\mathbb{C}}$ and $\left(x_{1}, x_{3}\right)$ extends continuously to $\left(x_{1}, x_{3}\right): \overline{\mathcal{R}} \rightarrow \widehat{\mathbb{C}}$ by taking $\left(x_{1}, x_{3}\right)(\infty)=\infty$. The pre-image of any point in $\left(x_{1}, x_{3}\right)(\mathcal{R})$ is a finite subset of $\mathcal{R}$, otherwise it would have an accumulation point at the boundary of $\mathcal{R}$, but $\partial G$ consists of monotone curves. Hence, $\left.\left(x_{1}, x_{3}\right)\right|_{\mathcal{R}}$ is a covering map of the simply connected $G$. 
Namely, $\left.\left(x_{1}, x_{3}\right)\right|_{\mathcal{R}}$ is injective. Therefore, $\left(x_{1}, x_{2}, x_{3}\right): \mathcal{R} \rightarrow \mathbb{R}^{3}$ is a graph.

Remark 6.1. The $U$-curve is not a symmetry curve. Otherwise, it would be in $O x_{1} x_{3}$ and the conjugate minimal surface would have a straight segment perpendicular to this plane. But $i d h$ is the 3 rd coordinate of the conjugate, and so its real part should be zero along the segment. However, in Section 4 we saw that $d h \notin \mathbb{R} \cup i \mathbb{R}$ along this curve.

6.3. Embeddedness proof. We shall some ideas from $[\mathbf{2 2}]$. Two copies of the fundamental domain are represented in Figure 16, together with corresponding $z$-image, namely two superimposed unitary disks.

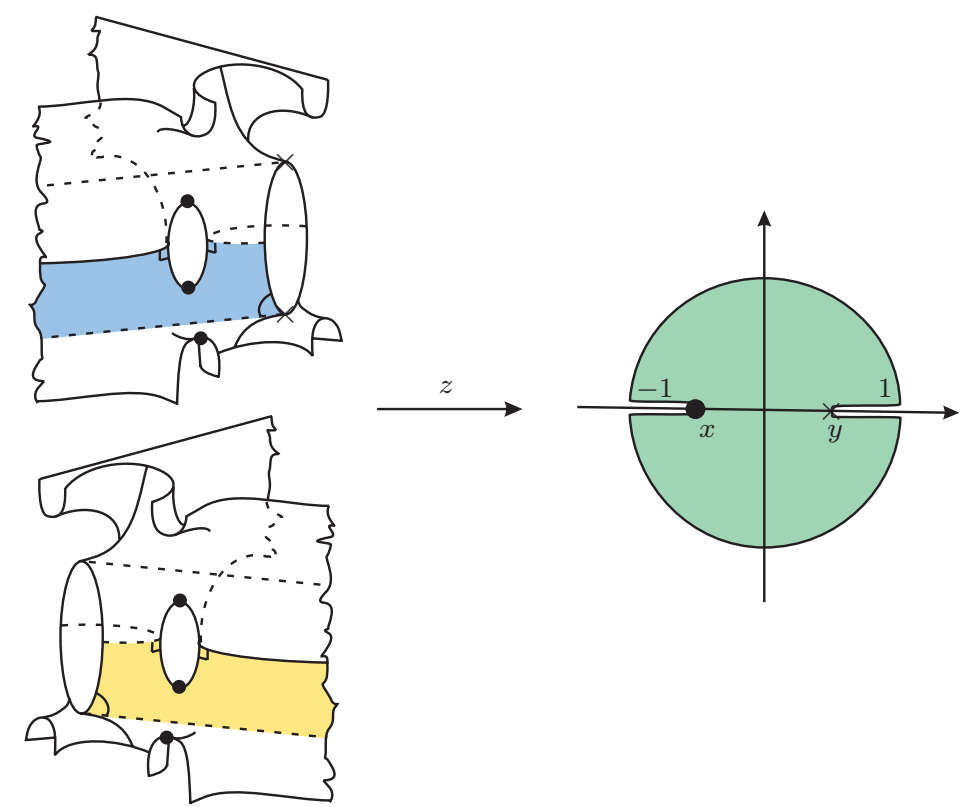

Figure 16. Two copies of the fundamental domain and their $z$-image.

The corresponding $g$-image is depicted in Figure 17. Let $\Gamma$ be the $z$-image of the $U$-curve. Then $\Gamma$ separates the superimposed disks into two disjoint components $\mathcal{A}$ and $\mathcal{B}$.

Figure 17 shows that $g(\mathcal{A})$ is the conjugate of $g(\mathcal{B})$, whereas $g(\Gamma)=$ $\widehat{\mathbb{R}} \backslash\{(-1,1)\}$. 

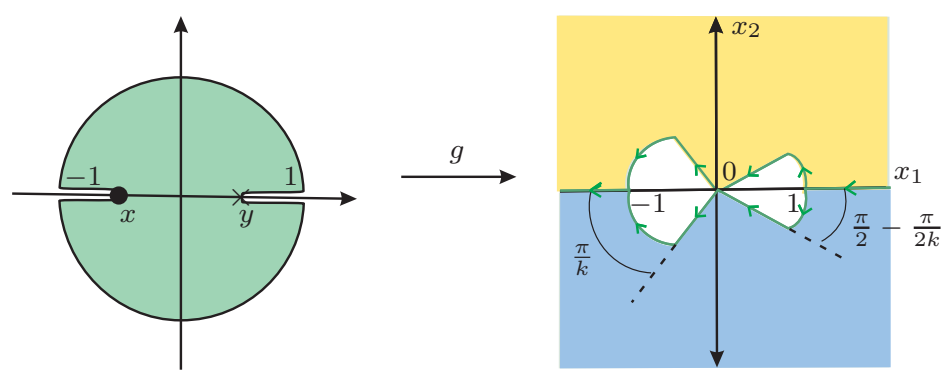

Figure 17 . The $g$-image of $\mathcal{A} \cup \mathcal{B}$.

In Subsection 6.2 we saw that $\left(x_{1}, x_{3}\right): \mathcal{A} \rightarrow \mathbb{R}^{2}$ and $\left(x_{1}, x_{3}\right): \mathcal{B} \rightarrow \mathbb{R}^{2}$ are both immersions. Again, Figure 15 shows the four possible features of $\left(x_{1}, x_{3}\right)(\mathcal{A})$. Since $d g$ never vanishes along the $U$-curve, then Figures $15(\mathrm{c})$ and $15(\mathrm{~d})$ cannot occur. We also discard Figure 15(b) by the same arguments presented in Subsection 6.2. So, there remains Figure $15(\mathrm{a})$ and we conclude that $\left(x_{1}, x_{2}, x_{3}\right): \mathcal{A} \rightarrow \mathbb{R}^{3}$ and $\left(x_{1}, x_{2}, x_{3}\right): \mathcal{B} \rightarrow$ $\mathbb{R}^{3}$ are both graphs.

Except for their boundaries, none of the graphs can intercept the other. Otherwise, they would either be tangent, or we could make them tangent by displacing both graphs in opposite directions along $O x_{2}$. This would contradict the Maximum Principle for Minimal Surfaces.

Therefore, both graphs intersect only at their coinciding $U$-curves, and what we have is an embedded double-piece of minimal surface contained in a wedge of $\mathbb{R}^{3}$. Its angle is $2 \pi / k$, and except for the $U$-curve, the double-piece has its rays and reflectional symmetry curves on the faces of the wedge. The whole $S T_{2 k}$ is then generated by successive rotations around the rays and reflections on the symmetry curves. Therefore, it has no self-intersections. Since $X$ is proper, then it is an embedding.

\section{References}

[1] F. Baginski and V. Ramos Batista, Solving period problems for minimal surfaces with the support function, Adv. Appl. Math. Sci. 9(1) (2011), 85-114.

[2] J. B. Conway, "Functions of one complex variable", Second edition, Graduate Texts in Mathematics 11, Springer-Verlag, New York-Berlin, 1978. 
[3] C. J. Costa, Example of a complete minimal immersion in $\mathbb{R}^{3}$ of genus one and three embedded ends, Bol. Soc. Brasil. Mat. 15(1-2) (1984), 47-54. DOI : $10.1007 /$ BF02584707.

[4] O. Forster, "Lectures on Riemann surfaces", Translated from the German by Bruce Gilligan, Graduate Texts in Mathematics 81, Springer-Verlag, New York-Berlin, 1981.

[5] L. Hauswirth, F. Morabito, and M. M. Rodríguez, An endto-end construction for singly periodic minimal surfaces, Pacific J. Math. 241(1) (2009), 1-61. DOI: 10.2140/pjm.2009.241.1.

[6] D. Hoffman And H. Karcher, Complete embedded minimal surfaces of finite total curvature, in: "Geometry, V", Encyclopaedia Math. Sci. 90, Springer, Berlin, 1997, pp. 5-93. DOI: 10.1007/978-3-662-03484-2_2.

[7] D. Hoffman And W. H. MeEks, III, Embedded minimal surfaces of finite topology, Ann. of Math. (2) 131(1) (1990), 1-34. DOI: 10.2307/1971506.

[8] L. P. Jorge And W. H. MeEks, III, The topology of complete minimal surfaces of finite total Gaussian curvature, Topology 22(2) (1983), 203-221. DOI : 10.1016/0040-9383(83)90032-0.

[9] H. Karcher, Embedded minimal surfaces derived from Scherk's examples, Manuscripta Math. 62(1) (1988), 83-114. DOI: 10.1007/ BF01258269.

[10] H. KARCHER, Construction of minimal surfaces, Surveys in Geometry, University of Tokyo (1989) and Lecture Notes 12, SFB256, Bonn (1989), available at http://www.math.uni-bonn.de/people/ karcher/karcherTokyo.pdf.

[11] F. J. López AND F. Martín, Complete minimal surfaces in $\mathbb{R}^{3}$, Publ. Mat. 43(2) (1999), 341-449. DOI: 10.5565/PUBLMAT_43299_01.

[12] F. J. López AND A. Ros, On embedded complete minimal surfaces of genus zero, J. Differential Geom. 33(1) (1991), 293-300.

[13] F. Martín and V. Ramos Batista, The embedded singly periodic Scherk-Costa surfaces, Math. Ann. 336(1) (2006), 155-189. DOI: $10.1007 / \mathrm{s} 00208-006-0778-z$.

[14] W. H. MeEks, III, J. PÉRez, And A. Ros, The geometry of minimal surfaces of finite genus III; bounds on the topology and index of classical minimal surfaces, Preprint, http://www . math.umass . edu/ bill/papers/24Sep2005.pdf.

[15] W. H. MeEks, III And M. Wolf, Minimal surfaces with the area growth of two planes: the case of infinite symmetry, J. Amer. Math. Soc. 20(2) (2007), 441-465. DOI : 10.1090/S0894-0347-06-00537-6. 
[16] J. C. C. Nitsche, "Lectures on minimal surfaces. Vol. 1. Introduction, fundamentals, geometry and basic boundary value problems", Translated from the German by Jerry M. Feinberg, With a German foreword, Cambridge University Press, Cambridge, 1989.

[17] R. Osserman, "A survey of minimal surfaces", Second edition, Dover Publications, Inc., New York, 1986.

[18] J. PÉREz AND M. TRAIZET, The classification of singly periodic minimal surfaces with genus zero and Scherk-type ends, Trans. Amer. Math. Soc. 359(3) (2007), 965-990 (electronic). DOI: 10.1090/S0002-9947-06-04094-3.

[19] V. Ramos Batista, A family of triply periodic Costa surfaces, Pacific J. Math. 212(2) (2003), 347-370. DOI: 10.2140/pjm. 2003. 212.347.

[20] A. H. SCHOEN, "Infinite periodic minimal surfaces without selfintersections", NASA technical note, no. D-5541, 1970.

[21] R. M. Schoen, Uniqueness, symmetry, and embeddedness of minimal surfaces, J. Differential Geom. 18(4) (1983), 791-809 (1984).

[22] M. F. DA Silva And V. Ramos Batista, Scherk saddle towers of genus two in $\mathbb{R}^{3}$, Geom. Dedicata 149 (2010), 59-71. DOI: 10.1007/s10711-010-9464-0.

[23] M. TRAizet, Construction de surfaces minimales en recollant des surfaces de Scherk, Ann. Inst. Fourier (Grenoble) 46(5) (1996), 1385-1442.

A. J. Yucra Hancco and G. A. Lobos:

DM, UFSCar

Rodovia Washington Luís, km 235

13565-905 São Carlos, SP

Brazil

E-mail address: alvaro@dm.ufscar.br

E-mail address: lobos@dm.ufscar.br

V. Ramos Batista:

CMCC, UFABC

Rua Santa Adélia 166, Bl.B

09210-170 Santo André, SP

Brazil

E-mail address: valerio.batista@ufabc.edu.br

Primera versió rebuda el 30 d'abril de 2013, darrera versió rebuda el 15 d'octubre de 2013. 